\title{
Fatores de risco para doenças sexualmente transmissíveis entre prostitutas e travestis de Ribeirão Preto (SP), Brasil
}

\author{
Afonso Dinis Costa Passos ${ }^{1}$ e José Fernando de Castro Figueiredo ${ }^{2}$
}

Como citar

Passos ADC, Figueiredo JF de C. Fatores de risco para doenças sexualmente transmissíveis entre prostitutas e travestis de Ribeirão Preto (SP), Brasil. Rev Panam Salud Publica. 2004;16(2):95-101.

RESUMO Objetivo. Caracterizar a população de profissionais do sexo em atividade em Ribeirão Preto, Brasil, segundo variáveis demográficas e socioeconômicas, e estudar os fatores de risco para doenças sexualmente transmissíveis nessa população.

Métodos. Foram visitados todos os locais de prática de prostituição identificados na cidade. Os participantes responderam um questionário que levantava informações sócio-demográficas e fatores de risco para doenças sexualmente transmissíveis. Foi responsável pelas entrevistas uma assistente social que há mais de 5 anos desenvolvia atividades educativas junto aos profissionais do sexo de Ribeirão Preto.

Resultados. As 449 prostitutas, 53 travestis e 13 michês estudados representam uma população jovem, de baixa escolaridade, reduzido nível socioeconômico e grande mobilidade espacial. Em relação às prostitutas, os travestis apresentaram risco significativamente mais elevado para doenças sexualmente transmissíveis, o qual se traduz por diferenças em termos do tempo de trabalho, número médio de parceiros por dia, antecedente de doenças sexualmente transmissíveis ulcerativas, prática de sexo anal, uso de drogas ilícitas não-injetáveis, especialmente o crack, e antecedente de prisão. A exposição a bebidas alcoólicas foi o único fator de risco mais freqüente entre as prostitutas. O uso de preservativo com parceiros fixos foi menos comum do que nas relações sexuais comerciais, tanto em prostitutas como em travestis.

Conclusões. Os profissionais do sexo de Ribeirão Preto, especialmente os travestis, representam uma população socialmente marginalizada e de alto risco para doenças sexualmente transmissíveis. É necessário que os serviços de saúde pública dediquem mais atenção aos profissionais do sexo, tanto em termos de programas preventivos como de realização de novas investigações que permitam um melhor conhecimento acerca dos fatores de risco específicos desse grupo para as doenças sexualmente transmissíveis.

Palavras-chave Comportamento sexual, profissional do sexo.

1 Universidade de São Paulo (USP), Faculdade de Medicina de Ribeirão Preto, Departamento de Medicina Social. Enviar correspondência para este autor no seguinte endereço: Avenida Bandeirantes 3900, CEP 14049-900, Ribeirão Preto, SP, Brasil. email: apassos@fmrp.usp.br

2 USP, Faculdade de Medicina de Ribeirão Preto, Departamento de Clínica Médica.
Os profissionais do sexo (PS) representam uma população de alto risco para as doenças sexualmente transmissíveis (DST) (1-6). Isso se deve a fatores diretamente ligados à prostituição tais como elevado número de parceiros e relações sexuais de risco - e a práticas e situações a ela associadas, aí incluídos o consumo de drogas ilícitas e de bebidas alcoólicas, a exposição a prisões, o baixo nível educacional e a marginalização socioeconômica $(1,2$, 
7-9). Por outro lado e, talvez, em parte devido ao estigma de que são vítimas, os PS têm recebido pouca atenção por parte dos órgãos de saúde pública e dos pesquisadores da área da saúde no Brasil, decorrendo daí uma grande carência de informações que permitam um melhor conhecimento de alguns aspectos ligados aos riscos a que são submetidos, essenciais para orientação de programas preventivos.

Ribeirão Preto não é uma exceção a essa regra. Com uma população de 500000 habitantes, constituindo-se em centro econômico-financeiro de uma das mais ricas regiões do Brasil, com cerca de 2 milhões de habitantes, a cidade tem atraído grandes fluxos migratórios de diferentes partes do país. Embora algumas instituições venham desenvolvendo trabalhos de apoio aos PS na cidade, nunca houve uma investigação sistematizada dessa população, que é desconhecida sob qualquer aspecto, ligado ou não à área da saúde.

Assim, o objetivo do presente estudo foi caracterizar a população de PS em atividade em Ribeirão Preto segundo variáveis demográficas e socioeconômicas, e estudar os fatores de risco para DST entre esses profissionais.

\section{MATERIAIS E MÉTODOS}

Para efeito de inclusão no trabalho foram utilizadas três categorias de PS, com as seguintes definições: 1) prostituta: mulher que pratica relação sexual predominantemente com homens, em troca de pagamento; 2 ) travesti: homem que trabalha travestido de mulher e que pratica relação sexual predominantemente com homens, em troca de pagamento; 3) michê: homem, geralmente jovem, que pratica relação sexual com pessoas de ambos os sexos, em troca de pagamento.

A população de PS de Ribeirão Preto foi inicialmente estimada em 400 a 500 indivíduos, com base em dados de duas instituições que prestam atendimento a essa população: a Secretaria de Saúde, através do seu Programa Municipal de Doenças Sexualmente Transmissíveis e AIDS (PMDSTAids), e a Universidade de São Paulo (USP), através do Núcleo de Estudos e Prevenção de Drogas e Aids (NEPDA). A participação dessas duas instituições possibilitou a identificação dos locais onde se encontravam os PS de Ribeirão Preto, como moradia ou local de trabalho, e o estabelecimento de um contato prévio. Todos os locais identificados foram visitados pela equipe, da qual fazia parte uma assistente social que há mais de 5 anos mantinha atividades educativas junto aos PS da cidade. Essa assistente social é ligada ao NEPDA e foi a responsável pelas entrevistas com os participantes. As visitas a cada local repetiram-se de modo a garantir que fossem contatados todos os indivíduos que nele residiam ou trabalhavam. Quando necessário, as visitas foram agendadas previamente, com o intuito de facilitar a localização dos potenciais participantes.

Quando se tratava de prostituição em áreas abertas (ruas, avenidas e praças) usaram-se, inicialmente, postos fixos próximos às áreas de trabalho dos PS, para os quais eles eram chamados a comparecer. Essas atividades foram complementadas com buscas ativas (nas quais sempre se fazia presente a assistente social) a pé ou de carro e com a participação de lideranças naturais, identificadas entre os potenciais participantes, que buscavam motivar os colegas a participar da investigação. Quando ocorreu o esgotamento dessa estratégia, passou-se a usar um ônibus especial, projetado para uso em pescarias, que possui poltronas, mesas e amplo espaço na parte traseira. Esse ônibus permitiu a inclusão de muitos PS que relutavam em deixar o seu local de trabalho e comparecer a um posto fixo. Outros potenciais participantes foram identificados e incluídos na pesquisa a partir de informações prestadas por pessoas que os conheciam, usando-se a técnica conhecida como "amostragem em bola de neve", ou snowball sample.

Após tomarem contato com os objetivos da pesquisa, os PS foram convidados a dela participar, após leitura e assinatura de um termo de consentimento. Um questionário padronizado foi aplicado a cada um dos participantes individualmente, visando levantar informações sócio-demográficas e fatores de risco para DST. Na mesma ocasião, uma amostra de $10 \mathrm{~mL}$ de sangue foi coletada de cada participante, com vistas a estudar a distribuição de marcadores sorológicos de hepatite B nessa população. O estrato social foi definido com base no critério de classificação econômica Brasil, utilizado pela Associação Nacional de Empresas de Pesquisa (10). Este critério é construído a partir das seguintes variáveis: grau de instrução do chefe da família, propriedade de alguns eletrodomésticos específicos e existência de empregada doméstica cujos serviços sejam pagos pelo participante. A cada um desses itens atribuem-se pontos, com o resultado sendo expresso numa classificação numérica descendente à qual se associam estratos: 25 a 34 pontos (estrato A), 17 a 24 pontos (estrato B), 11 a 16 pontos (estrato $C$ ), 6 a 10 pontos (estrato D) e 0 a 5 pontos (estrato E).

O teste do qui-quadrado $\left(\chi^{2}\right)$ foi empregado para testar associações entre as variáveis. Nas situações em que o seu uso foi inviabilizado, em virtude do pequeno número de participantes, utilizou-se o teste exato de Fisher.

A investigação foi aprovada pelo Comitê de Ética em Pesquisa do Hospital das Clínicas da Faculdade de Medicina de Ribeirão Preto, da USP. Os dados foram coletados entre agosto de 1999 e março de 2000.

\section{RESULTADOS}

Ao todo foram estudados 515 PS, representados por 449 prostitutas, 53 travestis e 13 michês (tabela 1). Embora a média da idade desses grupos tenha sido baixa e praticamente igual entre si, verificaram-se diferenças nas amplitudes de variação, com a mais larga delas ocorrendo entre as prostitutas (13 a 64 anos) e a menor entre os michês (18 a 29 anos). A observação dos locais de nascimento evidencia percentuais reduzidos de prostitutas e travestis nascidos em Ribeirão Preto e região, com mais da metade deles sendo originários de outros estados do país. Chama a atenção o fato de que menos da metade dos indivíduos nes- 
TABELA 1. Características sociais e demográficas de profissionais do sexo de Ribeirão Preto (SP), Brasil, 1999 a 2000

\begin{tabular}{|c|c|c|c|c|c|c|}
\hline \multirow[t]{2}{*}{ Característica } & \multicolumn{2}{|c|}{$\begin{array}{c}\text { Prostitutas } \\
(n=449)\end{array}$} & \multicolumn{2}{|c|}{$\begin{array}{l}\text { Michês } \\
(n=13)\end{array}$} & \multicolumn{2}{|c|}{$\begin{array}{l}\text { Travestis } \\
(n=53)\end{array}$} \\
\hline & \multirow{3}{*}{\multicolumn{2}{|c|}{$\begin{array}{c}23 \\
13 \text { a } 64\end{array}$}} & & & & \\
\hline Média & & & \multirow{2}{*}{\multicolumn{2}{|c|}{$\begin{array}{c}22 \\
18 \text { a } 29\end{array}$}} & \multirow{2}{*}{\multicolumn{2}{|c|}{$\begin{array}{c}23 \\
17 \text { a } 39\end{array}$}} \\
\hline Variação & & & & & & \\
\hline Local de nascimento & No. & $\%$ & No. & $\%$ & No. & $\%$ \\
\hline Ribeirão Preto & 28 & 6,2 & 6 & 46,1 & 5 & 9,5 \\
\hline Região de Ribeirão Preto & 37 & 8,3 & 1 & 7,7 & 6 & 11,3 \\
\hline Estado de São Paulo & 107 & 23,8 & 2 & 15,4 & 12 & 22,6 \\
\hline Outro estado & 277 & 61,7 & 4 & 30,8 & 30 & 56,6 \\
\hline \multicolumn{7}{|l|}{ Local de residência } \\
\hline Ribeirão Preto & 196 & 43,6 & 12 & 92,3 & 23 & 43,4 \\
\hline Região de Ribeirão Preto & 38 & 8,5 & - & - & 2 & 3,8 \\
\hline Estado de São Paulo & 64 & 14,3 & - & - & 11 & 20,8 \\
\hline Outro estado & 151 & 33,6 & 1 & 7,7 & 17 & 32,0 \\
\hline \multicolumn{7}{|l|}{ Escolaridade } \\
\hline$<2$ anos & 28 & 6,2 & 2 & 15,4 & 4 & 7,5 \\
\hline 2 a 4 anos & 54 & 12,0 & 2 & 15,4 & 4 & 7,5 \\
\hline 4 a 8 anos & 277 & 61,7 & 5 & 38,4 & 30 & 56,6 \\
\hline Ensino médio & 77 & 17,2 & 3 & 23,1 & 13 & 24,6 \\
\hline Universidade & 13 & 2,9 & 1 & 7,7 & 2 & 3,8 \\
\hline \multicolumn{7}{|l|}{ Estrato sociala ${ }^{\mathrm{a}}$} \\
\hline A & 2 & 0,5 & - & - & 1 & 1,9 \\
\hline$B$ & 41 & 9,1 & 2 & 15,4 & 13 & 24,5 \\
\hline C & 168 & 37,4 & 7 & 53,8 & 17 & 32,1 \\
\hline $\mathrm{D}$ & 180 & 40,1 & 2 & 15,4 & 15 & 28,3 \\
\hline$E$ & 58 & 12,9 & 2 & 15,4 & 7 & 13,2 \\
\hline Total & 449 & 100,0 & 13 & 100,0 & 53 & 100,0 \\
\hline
\end{tabular}

ses dois grupos reside em Ribeirão Preto e que aproximadamente um terço deles mantém residência em outros estados. Nas três categorias, predominou o nível de escolaridade entre 4 e 8 anos, seguido daqueles que chegaram a iniciar ou concluíram o ensino médio. Destacam-se percentuais consideráveis de indivíduos com escolaridade igual ou inferior a 4 anos, que variaram de $15 \%$, entre os travestis, a $30,8 \%$ entre os michês. Os estratos sociais $\mathrm{C}$ e $\mathrm{D}$ foram os mais observados nas três categorias, tendo atingido $77,5 \%$ entre as prostitutas.

Muito embora os michês se caracterizem por ter entre seus clientes indivíduos de ambos os sexos, chamou a atenção, na população do presente estudo, a constatação de que praticamente a totalidade deles referia atender apenas mulheres, podendo assim ser vistos como o equivalente masculino da prostituta. Além disso, a investigação de marcadores sorológicos de hepatite $B$, realizada em todos os participantes, mostrou valores de prevalência praticamente idênticos entre os michês e as prostitutas. Por essas razões, e tendo-se em conta o reduzido número de michês incluídos neste trabalho, optou-se por agrupá-los às prostitutas para efeito de comparação dos fatores de risco para DST (tabela 2), passando o grupamento a ser identificado como "prostitutas".

$\mathrm{O}$ único fator de risco que se mostrou mais presente no grupo das prostitutas foi a sensação de alcoolização, referida como ocorrendo diariamente ou ao menos uma vez por semana por $62,1 \%$, contra $34,6 \%$ dos travestis. Em contrapartida, os travestis mostraram proporções significativamente mais elevadas de presença dos demais fatores de risco, com exceção apenas de história de uso de drogas ilícitas intravenosas e de relação sexual com usuários de tais drogas, onde não se verificaram diferenças entre as duas categorias. No que diz respeito ao tempo de trabalho como PS, $58,2 \%$ das prostitutas haviam iniciado sua prática até 2 anos antes, proporção que alcançou apenas $15,1 \%$ entre os travestis. Um número médio diário de parceiros igual ou superior a três foi descrito por $48 \%$ das prostitutas e por $69,8 \%$ dos travestis. A comparação entre as duas categorias mostrou ainda diferenças significativas em relação aos seguintes antecedentes: DST de natureza ulcerativa, relação sexual anal, uso de drogas ilícitas não-intravenosas, uso de crack $(16,9$ e $39,6 \%)$, ter estado preso e uso constante de preservativos em relações sexuais de natureza comercial.

Embora não mostrado nas tabelas, merece registro o fato de que 226 prostitutas e 10 travestis relataram ter parceiros sexuais fixos, e o fato de que 71,6 e $28,6 \%$, respectivamente, referiram não usar preservativo nesse tipo de relação, ou usá-lo apenas eventualmente (diferença não significativa do ponto de vista estatístico).

\section{DISCUSSÃO}

Uma das maneiras de atenuar as dificuldades de acesso a populações que vivem em um estado de semiclandestinidade é a incorporação à pesquisa de pessoas que gozem da confiança dos potenciais participantes. Inquestionavelmente, o sucesso na inclusão de um número tão expressivo de profissionais do sexo neste trabalho deveu-se à participação ativa de uma assistente social familiarizada com a população alvo, que cumpriu o papel que a literatura define como "entrevistador de acesso privilegiado" (11). Além disso, teve grande importância a ajuda prestada por algumas prostitutas que espontaneamente colaboraram na arregimentação de participantes, exercendo o papel de informantes chaves, ou seja, indivíduos da própria comunidade, com reconhecida capacidade de 
TABELA 2. Fatores de risco para doenças sexualmente transmissíveis em prostitutas e travestis de Ribeirão Preto (SP), Brasil, 1999 a 2000

\begin{tabular}{|c|c|c|c|c|c|}
\hline \multirow[b]{2}{*}{ Fator de risco } & \multicolumn{2}{|c|}{$\begin{array}{l}\text { Prostitutas }^{a} \\
(n=462)\end{array}$} & \multicolumn{2}{|c|}{$\begin{array}{l}\text { Travestis } \\
(n=53)\end{array}$} & \multirow[b]{2}{*}{$P$} \\
\hline & No. & $\%$ & No. & $\%$ & \\
\hline \multicolumn{6}{|l|}{ Tempo de trabalho } \\
\hline$<6$ meses & 111 & 24,0 & 1 & 1,9 & \\
\hline 6 a 24 meses & 158 & 34,2 & 7 & 13,2 & \\
\hline$>24$ meses & 193 & 41,8 & 45 & 84,9 & $>0,0001$ \\
\hline \multicolumn{6}{|c|}{ Número médio de parceiros por dia } \\
\hline Até 2 & 240 & 52,0 & 16 & 30,2 & \\
\hline 3 a 5 & 189 & 40,9 & 28 & 52,8 & \\
\hline$>5$ & 33 & 7,1 & 9 & 17,0 & 0,0028 \\
\hline \multicolumn{6}{|c|}{$\begin{array}{l}\text { Antecedente de doença sexualmente } \\
\text { transmissível ulcerativa }\end{array}$} \\
\hline Sim & 52 & 11,3 & 16 & 30,2 & \\
\hline Não & 410 & 88,7 & 37 & 69,8 & 0,0003 \\
\hline \multicolumn{6}{|l|}{ Sexo anal } \\
\hline Sim & 52 & 11,3 & 53 & 100,0 & \\
\hline Não & 410 & 88,7 & - & - & $>0,0001$ \\
\hline \multicolumn{6}{|c|}{ Uso de drogas intravenosas ilícitas } \\
\hline Sim & 16 & 3,5 & 1 & 1,9 & \\
\hline Não & 446 & 96,5 & 52 & 98,1 & 0,8395 \\
\hline \multicolumn{6}{|c|}{ Sexo com usuário de drogas intravenosas } \\
\hline Sim & 96 & 20,8 & 15 & 28,3 & \\
\hline Não & 366 & 79,2 & 38 & 71,7 & 0,2779 \\
\hline \multicolumn{6}{|c|}{ Uso de drogas ilícitas não-intravenosas } \\
\hline Sim & 234 & 50,6 & 41 & 77,4 & \\
\hline Não & 228 & 49,4 & 12 & 22,6 & 0,0004 \\
\hline \multicolumn{6}{|l|}{ Consumo de crack } \\
\hline Sim & 78 & 16,9 & 21 & 39,6 & \\
\hline Não & 384 & 83,1 & 32 & 60,4 & 0,0001 \\
\hline \multicolumn{6}{|l|}{ Sentir-se sob efeito do álcool } \\
\hline Diária/semanalmente & 287 & 62,1 & 18 & 34,6 & \\
\hline Raro/nunca & 175 & 37,9 & 34 & 65,4 & 0,0002 \\
\hline \multicolumn{6}{|l|}{ Antecedente de ter sido preso } \\
\hline Sim & 59 & 12,8 & 24 & 45,3 & \\
\hline Não & 403 & 87,2 & 29 & 54,7 & $>0,0001$ \\
\hline \multicolumn{6}{|c|}{ Uso de preservativo em relação comercial } \\
\hline Sempre & 426 & 92,2 & 39 & 73,6 & \\
\hline Freqüente & 36 & 7,8 & 14 & 26,4 & $>0,0001$ \\
\hline Total & 462 & 100,00 & 53 & 100,00 & \\
\hline
\end{tabular}

a Inclui 449 prostitutas e 13 michês.

liderança e potencial para influenciar outros em relação a participar ou não da investigação (12).

Também foi decisiva a participação, no planejamento da investigação, das duas instituições que trabalham com os PS de Ribeirão Preto. Ambas têm longa tradição de contato próximo com essa população, que se traduz por um conhecimento detalhado dos locais de prática de prostituição na cidade e pela identificação da maioria dos envolvidos, a quem visitam com regularidade, prestam atendimento em termos das necessidades mais urgentes e distribuem preservativos. Este trabalho englobou todos os locais de prática de prostituição identificados como tais pelas duas instituições, aí incluídos pensões, hotéis, bordéis e ruas. Assim, tendo-se em conta as expectativas do pessoal do PMDSTAids e do NEPDA, as 449 prostitutas e os 53 travestis incluídos nesta investigação devem estar muito próximos do total de indivíduos de cada uma dessas categorias à época da realização da pesquisa. A busca intensiva dos PS e a inclusão da grande maioria daqueles identificados como tais conferem a este trabalho um cará- ter de pesquisa de base populacional. Minimiza-se, assim, a probabilidade de ocorrência de vieses de seleção, presentes quando a população de estudo é representada por grupos específicos, tais como pacientes de clínicas de tratamento de DST, ou a população de PS de uma única região da cidade (3). Além disso, reduz-se a possibilidade de que a proporção menor de travestis $(10,3 \%)$ na população decorra do fato de ser este um grupo de mais difícil identificação e acesso. Tudo indica que a participação majoritária das prostitutas $(87,2 \%)$ deva refletir, em grande parte, a distribuição dos PS em atividade em Ribeirão Preto, onde a maioria é representada por mulheres. Entretanto, deve-se reconhecer que o reduzido percentual de michês na população de estudo ( $2,5 \%$ do total) devese, muito provavelmente, às dificuldades de identificar este subgrupo.

Neste estudo, a idade média das prostitutas aproximou-se de 25 anos, sendo semelhante à média verificada em Glasgow, na Escócia (1), e inferior à média descrita em outros locais: 27 em Amsterdã, na Holanda (4); 27,2 no porto de Santos, Brasil (13); 27,6 em Londres, Inglaterra (14); e 29,8 na Espanha (2). A larga amplitude na idade das prostitutas deveu-se à presença de meninas de 13 anos entre as 16 prostitutas menores de idade incluídas, ao mesmo tempo em que houve uma participante com 64 anos.

O grande poder da Cidade de Ribeirão Preto de atrair os PS pode ser evidenciado pela constatação de que a maioria deles nasceu fora da cidade e da sua região de influência. Isso também é confirmado pelo fato de que um número significativo mantém residência oficial em outras cidades do estado ou em outras regiões do país, de modo particular em Minas Gerais e, secundariamente, no Paraná e em Goiás. Apesar de residirem fora da cidade, essas pessoas dirigem-se periodicamente a Ribeirão Preto onde permanecem por períodos variáveis, praticando a prostituição, antes de retornarem aos locais onde residem. A proximidade geográfica e a idealização de Ribeirão Preto como um eldorado econômico - conhecido em 
todo o país como a "Califórnia brasileira" - , devem ser os fatores mais relevantes para explicar esses achados. Do ponto de vista sanitário, essa rotatividade representa um obstáculo considerável à implementação e sustentação de programas preventivos, além de possibilitar a introdução de agentes infectantes em áreas indenes.

A constatação de que mais de $70 \%$ dos participantes tinham escolaridade máxima de 8 anos - com cerca de $18 \%$ deles com não mais de 4 anos evidencia o reduzido grau de educação da população estudada. A título de comparação, os dados do censo brasileiro realizado no ano de 2000 mostram que 59,6\% do total de habitantes de Ribeirão Preto tinham escolaridade máxima de 8 anos (15). Esse nível mais reduzido de escolaridade entre os PS não pode ser imputado à baixa idade dos participantes, uma vez que apenas 16 deles eram menores de 18 anos. Além disso, mesmo o mais jovem já havia completado o seu $13^{\circ}$ aniversário; portanto, em condições normais, esperava-se dele uma freqüência mínima de 6 anos à escola. Portanto, a reduzida escolaridade da maioria dos participantes deve ter resultado das dificuldades de acesso à escola, ou, mais provavelmente, do abandono precoce do estudo. Dados de um levantamento entre prostitutas atuando em Londres apontam para a segunda hipótese, na medida que revelam percentual elevado de abandono da escola já entre indivíduos muito jovens, atingindo $62 \%$ até os 16 anos de idade (16).

O predomínio dos estratos sociais C e D demonstra que, no geral, a população estudada tem condição socioeconômica desfavorável, evidenciada quando se comparam esses dados com a distribuição dos mesmos estratos na população geral brasileira (10). O estrato A, correspondente a $5 \%$ do total de habitantes do País, inclui apenas $0,6 \%$ dos participantes deste estudo. No estrato B, o valor para o Brasil (19\%) também é bastante superior ao da população estudada (10,9\%). Em contrapartida, os participantes concentram-se nos estratos C (37,3\%), D $(38,3 \%)$ e E (13\%), para os quais a população brasileira apresenta valores respectivos de 31, 33 e 12\%. A comparação com a Região Sudeste é ainda mais desfavorável aos participantes, de modo particular no estrato $B$, onde a região concentra $22 \%$ dos seus habitantes, contra $10,9 \%$ dos PS, e no estrato $\mathrm{E}$, onde os valores são de $6 \%$ e $13 \%$, respectivamente.

Como as idades médias das prostitutas e dos travestis foram muito próximas entre si, o achado de que o segundo grupo concentra indivíduos com mais tempo de trabalho somente pode ser explicado por um início mais precoce das suas atividades como PS. Somado a um número médio maior de parceiros por dia, à prática corrente de sexo anal e à constatação de que os participantes deste grupo relatam, com mais freqüência, falhas no uso de preservativos nas suas relações comerciais, verifica-se que os travestis apresentam um risco muito mais elevado do que as prostitutas de desenvolverem DSTs, uma vez que sofrem um efeito cumulativo de exposições potencialmente capazes de transmitir essas doenças. Isso é parcialmente confirmado pela constatação de antecedentes de DST ulcerativa em 30,2\% dos travestis, contra $11,3 \%$ das prostitutas. Nesse caso, tal tipo de doença não só indica a presença pregressa de riscos diferenciados, como também representa, ela própria, um risco adicional para se adquirir DSTs, uma vez que doenças genitais ulcerativas são condições facilitadoras da entrada de agentes infecciosos (4). Além dos fatores anteriormente mencionados, os travestis diferiram das prostitutas também no que diz respeito a maiores proporções de uso de drogas ilícitas não-intravenosas, particularmente crack, e história de prisão pregressa.

Em relação ao uso de drogas ilícitas, chama a atenção o reduzido percentual de uso, atual ou passado, de produtos injetáveis, tanto entre prostitutas $(3,5 \%)$ como entre travestis $(1,9 \%)$. E mais: com exceção de apenas uma prostituta que se declarou usuária regular, os demais participantes referiram uso eventual e esporádico de drogas injetáveis. Embora, em virtude do estigma associado a esse comportamento, não se possa descartar por completo a possibilidade de respostas falsamente negativas a essa questão, a relação de conhecimento e familiaridade da entrevistadora com os participantes leva a crer que, se tais respostas ocorreram, seu número foi desprezível. Este achado contrasta com algumas referências da literatura que apontam para um forte consumo de drogas ilícitas injetáveis entre PS, com percentuais de até $81 \%$ na Escócia (1) e 82\% na Holanda (4).

Em contrapartida ao reduzido uso de drogas injetáveis, observaram-se proporções acima de $20 \%$ de antecedentes de relacionamento sexual com usuários de tais drogas. Tais números devem ser vistos com cautela, uma vez que refletem o conhecimento do PS acerca da prática de consumo de drogas por parte do seu parceiro. Como a condição de usuário de drogas ilícitas injetáveis nem sempre é perceptível a um contato sexual de ocasião, é possível que esses percentuais sejam mais elevados do que os referidos pelos participantes. Este fato assume importância sanitária considerável, uma vez que os riscos de transmissão de DSTs ocorrem também no sentido do cliente para o PS e não apenas no sentido inverso, tal como geralmente aceito (17).

Nesse particular, merece destaque o achado de elevadas proporções de PS que não usam ou fazem uso irregular de preservativo nas suas relações sexuais com parceiros fixos, fato que confirma observações realizadas no Brasil (5) e em inúmeros países (1, 4, $11,14,16-19)$. Isso talvez reflita a confiança - muita vezes irrestrita - e o sentimento de que o uso do preservativo tende a caracterizar a relação sexual como comercial, algo que se faz em troca de dinheiro, e não com o parceiro com o qual se mantém algum vínculo afetivo (16).

Diferentemente das drogas ilícitas injetáveis, os antecedentes de consumo de drogas ilícitas não-injetáveis foram referidos pela metade das prostitutas e por quase $80 \%$ dos travestis, corroborando as altas proporções descritas entre PS de diferentes partes do mundo $(2,11,14,18)$. As drogas nãoinjetáveis referidas pelos participantes 
englobaram, fundamentalmente, maconha, cocaína e crack, usadas de modo isolado ou combinado. Dentre essas, a de maior relevância para a transmissão de DSTs é, inquestionavelmente, o crack, em função de sua grande capacidade de produzir dependência, em parte porque age rapidamente sobre o sistema nervoso central produzindo uma euforia intensa e fugaz, seguida por depressão profunda e necessidade compulsiva de voltar a consumir a droga $(7,20)$.

Do ponto de vista econômico, a forte dependência causada pelo crack torna a sua demanda inelástica em relação ao seu preço e à renda do usuário (8). Assim, difere das drogas consideradas como "recreacionais", tais como o tabaco e a maconha, cuja elasticidade de demanda reflete-se na redução do consumo como resposta à eventual elevação de preço ou à queda da renda do usuário. Por ter demanda inelástica em relação ao custo e à capacidade aquisitiva do dependente, o consumo do crack tende a ser mantido mesmo que à custa de privações de toda a ordem (8). Essa condição torna os PS vulneráveis, muitas vezes obrigados a reduzir o preço e a aumentar o número de programas, além de se sujeitarem a práticas de alto risco exigidas pelos parceiros. Em casos mais extremos, a prostituição passa a ser realizada com a finalidade quase que única de obter a droga, passando as outras necessidades a ocupar plano secundário no rol das prioridades cotidianas $(8,21)$. Dramática de qualquer ponto de vista, essa situação foi encontrada entre participantes deste trabalho, tendo sido verificadas situações em que o preço cobrado era de $\mathrm{R} \$ 2,00$ pelo programa (equivalente a US\$1,5 na época da pesquisa), suficiente apenas para comprar a próxima pedra de crack. A detenção em delegacias ou prisões é outro fator de risco conhecido para DSTs (9), tanto em decorrência de há- bitos e estilos de vida prévios, como de exposições que passam a ocorrer após o encarceramento. Os elevados percentuais de detenção, particularmente entre os travestis, denotam a desagregação social e as perseguições de que são vítimas esses indivíduos, muitas vezes detidos em arrastões policiais de rua, mesmo que nenhuma prática delituosa seja caracterizada (o código penal brasileiro caracteriza como crime apenas a exploração da prostituição, e não a sua prática).

Chama a atenção o fato de que aproximadamente dois terços das prostitutas e um terço dos travestis referiam alcoolização diária ou freqüente (ao menos 1 vez por semana), constituindo essa a única exposição a um potencial fator de risco que se fez presente em proporção mais elevada no grupo das prostitutas. O consumo elevado de bebidas alcoólicas por PS parece ser uma constante em diferentes países do mundo, embora os valores observados entre as prostitutas de Ribeirão Preto superem os descritos para as prostitutas de rua de Londres $(11,14)$ e de Nova Iorque (18). Superam também, por larga margem, o consumo pelos travestis incluídos nesta investigação. Esses achados devem ser decorrentes do grande número de prostitutas que trabalhavam em chácaras, boates e pequenas pensões e hotéis, onde uma das funções esperadas das PS é levar os seus parceiros a consumir a maior quantia possível de bebidas alcoólicas, uma vez que na venda dessas substâncias reside uma parte substancial dos lucros da casa. Muitos desses locais estabelecem uma quota semanal mínima de doses, cujo consumo é cobrado de cada uma das prostitutas que ali trabalham. Isso leva a PS a induzir o cliente a gastar mais em bebidas para o consumo de ambos, atingindo-se mais facilmente a quota estabelecida. Além desse fato, puramente comercial, outra razão apontada para o elevado consumo de bebidas alcoólicas são dificuldades inerentes à prostituição, as quais seriam mais facilmente enfrentadas quando o PS usa alguma substância com efeito desinibitório, caso do álcool e de outras drogas $(11,14)$. É justamente em decorrência desse efeito que o consumo de bebidas alcoólicas tem sido associado à maior ocorrência de sexo inseguro entre prostitutas (11, 14) e travestis (22).

Os dados deste trabalho evidenciam que os PS de Ribeirão Preto compõem uma população jovem, de baixa escolaridade e reduzido nível socioeconômico, oriunda em grande parte de outras cidades do Estado de São Paulo e de outros estados, e com grande mobilidade espacial. Associando-se a isso, verifica-se a presença de outros comportamentos de risco de grande relevância, particularmente exposição a drogas, alcoolismo e antecedentes de prisão. Tais características não diferem, na sua essência, daquelas de PS de diferentes países do mundo. Tradicionalmente marginalizados pela sociedade, constituem, quase sempre, grupos populacionais de baixo poder aquisitivo, muitas vezes vivendo em aguda situação de pobreza, obrigados a constantes deslocamentos em busca de mercado mais favorável $(1,2,18)$ e expostos a práticas que os colocam em risco constante para DSTs. Confirmando outras observações (23), isso é ainda mais visível para o grupo de travestis, vítimas de uma carga maior de estigma e marginalização social, que contribui para torná-los mais arredios às tentativas de contato (6). Torna-se, assim, necessário que os serviços de saúde pública passem a dedicar mais atenção aos PS, tanto do ponto de vista de oferta de programas preventivos, como da perspectiva de desenvolvimento de novas investigações que permitam um melhor conhecimento acerca dos fatores de risco específicos desse grupo para as DSTs. 
1. Green ST, Goldberg DJ, Christie PR, Frischer M, Thomson A, Carr SV, et al. Female streetworker-prostitutes in Glasgow: a descriptive study of their lifestyle. AIDS Care. 1993;5(3): 321-35.

2. Estébanez P, Zunzunegui V, Aguillar D, Coloma C, Rua-Figueroa M, Fitch K, et al. A demographic and health survey of Spanish female sex workers: HIV prevalence and associated risk factors. J Biosoc Sci. 1998;30(3): 365-79.

3. Tabet SR, Palmer DL, Wiese WH, Voorhees RE, Pathak DR. Seroprevalence of HIV-1 and hepatitis $\mathrm{B}$ and $\mathrm{C}$ in prostitutes in Albuquerque, New Mexico. Am J Public Health. 1992; 82(8):1151-4.

4. Van den Hoek JAR, van Haastrecht HJA, Scheeringa-Troost B, Goudsmit J, Coutinho RA. HIV infection and STD drug addicted prostitutes in Amsterdam: potential for heterosexual HIV transmission. Genitourin Med. 1989; 65(3):146-50.

5. Lurie P, Fernandes MEL, Hughes V, Arevalo EI, Hudes ES, Reingold A, et al. Socioeconomic status and risk of HIV-1, syphilis and hepatitis B infection among sex workers in São Paulo State, Brazil. AIDS. 1995;9(Suppl 1): S31-7.

6. Elifson KW, Boles J, Posey E, Sweat M, Darrow W, Elsea W. Male transvestite prostitutes and HIV risk. Am J Public Health. 1993;83(2): 260-2.

7. Jones DL, Irwin KL, Inciardi J, Bowser B, Schilling $\mathrm{R}$, Word $\mathrm{C}$, et al. The high-risk sexual practices of crack-smoking sex workers recruited from the streets of three American cities. Sex Transm Dis. 1998;25(4):18793.
8. Baseman J, Ross M, Williams M. Sale of sex for drugs and drugs for sex: an economic context of sexual risk behavior for STDs. Sex Transm Dis. 1999;26(8):444-9.

9. Singh S, Prasad R, Mohanty A. High prevalence of sexually transmitted and blood-borne infections amongst the inmates of a district jail in Northern India. Int J DST AIDS. 1999; 10(7):475-8.

10. Associação Nacional de Empresas de Pesquisa. Critério de classificação econômica Brasil. São Paulo: ANEP; 2003. Disponível em: www.anep.org.br/codigosguias/CCEB.pdf Acessado em 17 de maio de 2004.

11. Gossop M, Powis B, Griffiths P, Strang J. Female prostitutes in south London: use of heroin, cocaine and alcohol, and their relationship to health risk behaviours. AIDS Care. 1995;7(3):253-60.

12. Watters JK, Biernacki P. Targeted sampling: options for the study of hidden populations. Social Problems. 1989;36(4):416-30.

13. Mesquita PE, Granato CFH, Castelo A. Risk factors associated with hepatitis $C$ virus (HCV) infection among prostitutes and their clients in the city of Santos, São Paulo State, Brazil. J Med Virol. 1997;51(4):338-43.

14. Gossop M, Powis B, Griffiths P, Strang J. Sexual behaviour and its relationship to drugtaking among prostitutes in south London. Addiction. 1994;89(8):961-70.

15. Instituto Brasileiro de Geografia e Estatística [site da Internet]. Disponível em: http:// www2.ibge.gov.br/pub/Censos/Censo Demografico_2000/educacao/ Acessado em maio de 2004.

16. Ward H, Day S, Mezzone J, Dunlop L, Donegan C, Farrar S, et al. Prostitution and risk of
HIV: female prostitutes in London. Br Med J. 1993; 307(6900):356-8

17. Day S, Ward H, Perrotta L. Prostitution and risk of HIV: male partners of female prostitutes. Br Med J. 1993;307(6900):359-62.

18. Witte SS, El-Bassel N, Wada T, Gray O, Wallace J. Acceptability of female condom use among women exchanging street sex in New York City. Int J STD AIDS. 1999;10(3):162-8.

19. Spina M, Mancuso S, Sinicco A, Vaccher E, Traina C, Di Fabrizio N, et al. Human immunodeficiency virus seroprevalence and condom use among female sex workers in Italy. Sex Transm Dis. 1998;25(9):451-4.

20. Edlin BR, Irwin KL, Faruque S, McCoy CB, Word C, Serrano Y, et al. Intersecting epidemics - crack cocaine use and HIV infection among inner-city young adults. N Engl J Med. 1994; 331(21):1422-7.

21. Ferri C, Gossop M. Route of cocaine administration: patterns of use and problems among a Brazilian sample. Addict Behav. 1999;24(6): 815-21.

22. Baqi S, Shah SA, Baig MA, Mujeeb SA, Memon A. Seroprevalence of HIV, HBV and syphilis and associated risk behaviours in male transvestites (Hijras) in Karachi, Pakistan. Int J DST AIDS. 1999;19(5):300-4.

23. Day S, Ward H. Sex workers and the control of sexually transmitted disease. Genitourin Med. 1997;73(3):161-8.

Manuscrito recebido em 7 de agosto de 2003. Aceito em versão revisada em 12 de março de 2004
ABSTRACT

Risk factors for sexually transmitted diseases in prostitutes and transvestites in Ribeirão Preto (SP), Brazil
Objective. To describe the demographic and socioeconomic characteristics of sex workers in the city of Ribeirão Preto, which is in the state of São Paulo, Brazil, and to investigate the risk factors for sexually transmitted diseases (STDs) in this population. Methods. All the areas in the city where female or male prostitutes or transvestites work or live were visited. The participants answered a questionnaire that collected sociodemographic data and information concerning risk factors for STDs. A social worker who for more than five years had carried out educational activities with sex workers in Ribeirão Preto was responsible for the interviews.

Results. The 449 female prostitutes, 13 male prostitutes, and 53 male transvestite sex workers who were included in the study make up a young population, with little schooling and a low socioeconomic level. A majority of them were born in states other than São Paulo, and a third of them still maintain a residence in some other state. In comparison to the prostitutes (the female and male prostitutes considered together), the transvestites had a significantly higher risk for STDs, which was reflected in their number of years as sex workers, average number of sexual partners per day, history of ulcerative STDs, practice of anal sex, use of illegal noninjectable drugs (especially crack), and history of incarceration. Exposure to alcohol was the only risk factor found more frequently in the prostitutes. Using a condom with a steady partner was less frequent than was condom use in commercial sex, for both the prostitutes and the transvestites. Conclusions. Sex workers in Ribeirão Preto, especially transvestites, are socially marginalized and at high risk for STDs. Public health services should focus more attention on this population by developing prevention programs and by supporting additional research that could provide more detailed knowledge concerning the specific risk factors that put this population at risk for STDs. 